\title{
NRG1 regulates redox homeostasis via NRF2 in papillary thyroid cancer
}

\author{
TING-TING ZHANG ${ }^{*}$, NING QU ${ }^{1 *}$, GUO-HUA SUN ${ }^{1 *}$, LONG ZHANG $^{2}$, YUAN-JIN WANG ${ }^{3}$, XIANG-MING MU ${ }^{3}$, \\ WEN-JUN WEI ${ }^{1}$, YU-LONG WANG ${ }^{1}$, YU WANG ${ }^{1}$, QING-HAI JI ${ }^{1}$, YONG-XUE ZHU ${ }^{1}$ and RONG-LIANG SHI ${ }^{1}$ \\ ${ }^{1}$ Department of Head and Neck Surgery, Fudan University Shanghai Cancer Center; ${ }^{2}$ Fudan University Shanghai Cancer Center \\ and Institutes of Biomedical Sciences, Collaborative Innovation Center of Cancer Medicine, Shanghai Medical College, \\ Fudan University, Shanghai 200032; ${ }^{3}$ Department of General Surgery, Yancheng First People's Hospital, \\ The Fourth Affiliated Hospital of Nantong Medical College, Yancheng, Jiangsu 224000, P.R. China
}

Received February 15, 2018; Accepted May 21, 2018

DOI: $10.3892 /$ ijo.2018.4426

\begin{abstract}
Thyroid cancer is a common endocrine cancer, of which papillary thyroid cancer (PTC) is the most common type. Neuregulin 1 (NRG1), a glycoprotein mediating cell-cell signaling, plays vital roles in cellular activities; however, its role in PTC progression remains poorly understood. In this study, we performed immunohistochemistry in 196 samples from patients and found that NRG1, a potential prognostic marker is highly expressed in PTC compared with adjacent normal tissues. Cell Counting kit-8 (CCK-8) and clone formation assays indicated that NRG1 is essential for PTC cell viability and proliferation, probably by regulating redox homeostasis, which was implied by ROS generation analysis and intracellular GSH activity assay. Western blot analysis and RT-qPCR revealed that NRG1 regulates ERK pathway and the pivotal regulator of cellular redox status, nuclear factor E2-related factor 2 (NRF2), which maintains moderate reactive oxygen species (ROS) levels through a set of antioxidant response element (ARE)-containing genes. The immunohistochemical scoring of 196 PTC samples and the analysis of the data of 490 patients from The Cancer Genome Atlas (TCGA) reveled a positive association between the expression of NRG1 and NRF2. Since the presence of NRG1 regulates redox homeostasis through NRF2, protecting PTC cells from the accumulation of ROS and ROS-induced cell death, NRG1 may thus prove to be a potential therapeutic target in the treatment of thyroid cancer.
\end{abstract}

Correspondence to: Professor Rong-Liang Shi or Professor Yong-Xue Zhu, Department of Head and Neck Surgery, Fudan University Shanghai Cancer Center, 270 Dongan Road, Shanghai 200032, P.R. China

E-mail: shirongliang@126.com

E-mail: zhongyongxue@shca.org.cn

${ }^{*}$ Contributed equally

Key words: neuregulin 1, nuclear factor E2-related factor 2, papillary thyroid cancer, redox homeostasis

\section{Introduction}

Papillary thyroid cancer (PTC) is the most common type of thyroid cancer. As epidemiological studies have reported, the incidence of thyroid cancer has markedly increasing over the past decades, mainly due to the increasing incidence of PTC (1). PTC typically has a favorable prognosis, with an overall 10-year survival rate $>90 \%$. However, the number of patients with refractory PTC has also increased, which is an obstacle to the effective treatment of PTC (2). Thus, the further understanding of the molecular mechanisms responsible for the development and progression of PTC is important in order to improve the prognosis (3).

Neuregulin 1 (NRG1) is one of the most active members of the epidermal growth factor (EGF)-like family, located on chromosome 8p12. NRG1 is a membrane glycoprotein that mediates cell-cell signaling and plays a critical role in the growth and development of multiple organ systems. $N R G 1$ is processed into numerous isoforms by alternative splicing, which allows it to perform a wide variety of functions $(4,5)$. The interaction of NRG1 with the dimers of its receptors, including ErbB2, ErbB3 and ErbB4, results in a number of biological processes (6). NRG1 has been reported to interact with several signal pathways, including the Ras/MAPK/ ERK1/2 pathway in Schwann cell development and the PI3K pathway in melanoma $(7,8)$.

To achieve uncontrolled proliferation, it is critical for tumor cells to maintain reduction and oxidation balance, which is also known as redox homeostasis. Reactive oxygen species (ROS) need to be regulated delicately to a moderate level so that tumor cells can initiate, proliferate, migrate and invade in a well-balanced redox homeostasis $(9,10)$. As byproducts of oxygen metabolism, ROS play critical physiological roles in biological processes and are important for healthy cell function (11). However, high ROS levels are noxious to cancer cells, as DNA damage can be induced by excessive ROS generation, which eventually leads to apoptosis $(12,13)$.

Cellular oxidative stress is aggravated when ROS levels increase, and tumor cells have to confront a severe survival challenge (12). In order to survive, tumor cells apply a series of strategies with which to restore redox homeostasis. For example, tumor cells activate complex antioxidant pathways 
to increase the expression of ROS scavengers, thus neutralizing excessive ROS levels (10). The transcription factor nuclear factor E2-related factor 2 (NRF2), is a pivotal regulator of a series of antioxidant enzymes. NRF2 upregulates the expression of these antioxidant enzymes by binding to the antioxidant response element (ARE) in their promoter regions (14). In non-stressed cells, NRF2 can be degraded by the constitutively active Kelch-like ECH-associated protein 1 (KEAP1)-mediated and cullin-3-dependent ubiquitin-proteasome pathway, which is disrupted upon oxidative stress, leading to NRF2 accumulation. NRF2 then translocates to the nucleus and triggers the antioxidant response (15).

However, to date, and at least to the best of our knowledge, there have been only a few attempts made at exploring the role of NRG1 in PTC (16-18). Thus, in the present study, we aimed to assess the role of NRG1 in PTC, particularly the association between NRG1 and PTC cell viability and proliferation. Furthermore, we aimed to elucidate the underlying mechanisms by exploring the potential role of NRG1 in the regulation of thyroid cancer-related pathways, including ERK1/2 and redox homeostasis.

\section{Materials and methods}

Patients and tissue samples. Following confirmation by pathological diagnosis, thyroid cancer tissues and corresponding normal thyroid tissues at least $1 \mathrm{~cm}$ away from the tumor were obtained from 196 patients. These patients were diagnosed and had received a thyroidectomy between 2003 and 2012 at the Department of Head and Neck Surgery, Fudan University Shanghai Cancer Center (Shanghai, China). All tissue samples were fixed in formalin, embedded in paraffin and sectioned into 5- $\mu \mathrm{m}$-thick slices. The criteria of the American Joint Committee on Cancer (8th edition) for thyroid cancer was used for TNM staging classification (19). This study was approved by the Human Ethics Committee/Institutional Review Board of Fudan University Shanghai Cancer Center. All 196 patients signed the written informed consent. A highly sensitive streptavidin-biotin-peroxidase detection system was used to perform immunohistochemical staining with thyroid cancer tissue microarrays derived from the samples of the 196 patients. To investigate the association between the expression of NRG1 and that of NRF2, we extracted and analyzed the data of 490 patients with PTC from The Cancer Genome Atlas (TCGA) database. NRG1 and NRF2 expression and clinical data of the TCGA database are available from the link directly: http://www.cbioportal.org/index.do?cancer_study_id= thca_tcga\&Z_SCORE_THRESHOLD=2.0\&RPPA_SCORE_ THRESHOLD $=2.0 \&$ data_priority $=0 \&$ case_set_id=thca_tcga_ all\&gene_list=NRG1\%2520NFE2L2\&geneset_list $=+\&$ tab_index $=$ tab_visualize\&Action=Submit\&genetic_profile_ids_PROFILE_ MUTATION_EXTENDED=thca_tcga_mutations\&genetic_ profile_ids_PROFILE_COPY_NUMBER_ALTERATION= thca_tcga_gistic. Patients who fit the following criteria were included: i) Patients who had PTC as the only malignancy or the first of multiple malignancies; and ii) patients who received surgical therapy. Patients who fit the following criteria were excluded: i) Patients with insufficient data or unknown clinicopathological profiles; ii) patients with an undetermined histology; and iii) patients with other types of thyroid cancer (follicular thyroid cancer, medullary thyroid cancer, anaplastic thyroid cancer, etc.), or secondary tumors.

Immunohistochemistry (IHC) and IHC scoring. Briefly, tissue samples obtained from the 196 patients were fixed with formalin (37\% formaldehyde dissolved in water, containing $10 \%$ methanol in addition) at room temperature for $24 \mathrm{~h}$. The fixed samples were then embedded in paraffin and sectioned into $5-\mu \mathrm{m}$-thick slices. The slices were mounted on slides and were then deparaffinized with xylol, hydrated with gradient ethanol in multiple steps. Citrate buffer ( $\mathrm{pH}$ 6.0) was used for antigen retrieval and the slides were heated in this buffer at $121^{\circ} \mathrm{C}$ for $15 \mathrm{~min}$. Following the blockage of endogenous peroxidase by $3 \% \mathrm{H}_{2} \mathrm{O}_{2}$, the slides were then blocked by $10 \%$ normal goat serum and incubated with primary antibodies. After being washed with PBS, the slides were developed using Dako EnVision + Rabbit Polymer (cat. no. K4003) from Dako (Carpinteria, CA, USA), followed by another thorough washing. The slides were counterstained with hematoxylin and coverslipped. The immunohistochemically-stained samples were scored by two pathologists blinded to the clinical parameters, separately. The staining intensity was scored as follows: 0 (negative), 1 (weak), 2 (medium) or 3 (strong). The extent of staining was scored as follows: $0,<5 \% ; 1,5-25 \% ; 2,26-50 \% ; 3$, $51-75 \%$; and $4,>75 \%$ according to the percentages of the positively stained areas in relation to the whole tumor area. The immunoreactivity score (IS) for each sample was generated by multiplying the score for staining intensity with the score for staining extent. Final staining scores for samples of $<4,4$, 6 and $\geq 8$ were considered to be,,-+++ and +++ , respectively.

Cell culture. Two human papillary thyroid cancer cell lines, $\mathrm{K} 1$ and TPC1, were purchased from the University of Colorado 125 Cancer Center Cell Bank and were cultured in RMPI-1640 medium containing 10\% FBS (Invitrogen, Carlsbad, CA, USA). 293T cells were purchased from the Type Culture Collection Cell Bank, Chinese Academy of Sciences and were cultured in Dulbecco's modified Eagle's medium containing 10\% FBS (Invitrogen). All cells were maintained at $37^{\circ} \mathrm{C}$ with $5 \% \mathrm{CO}_{2}$ in proper humidity. It should be noted that the $\mathrm{K} 1$ cells are considered to be a mixed thyroid gland papillary carcinoma type, as it has been reported that the $\mathrm{K} 1$ cells are contaminated by GLAG-66, which is also derived from thyroid gland papillary carcinoma $(20,21)$.

Antibodies. NRG1 antibody (10527-1-AP), NRF2 antibody (16396-1-AP), GAPDH (60004-1-Ig) and ERK1/2 antibody (16443-1-AP) were purchased from Proteintech Group, Inc. (Chicago, IL, USA) $\beta$-actin antibody (sc-47778) was obtained from Santa Cruz Biotechnology (Santa Cruz, CA, USA). The secondary antibodies, anti-mouse (SA00001-1) antibody conjugated with horseradish peroxidase (HRP) and anti-rabbit (SA00001-2) antibody conjugated with HRP, were purchased from Proteintech Group, Inc. All antibodies were diluted as per the manufacturer's instructions when they were applied for specific experiments. NRG1 antibody and NRF2 antibody were diluted at a ratio of 1:50 for IHC and were diluted 1:1,000 for western blot analysis. GAPDH antibody and ERK1/2 antibody were diluted at a ratio of 1:1,000 for western blot analysis. $\beta$-actin antibody was diluted 1:500 for western blot analysis. 
Plasmids. The pLKO.1-TRC cloning vector (Addgene plasmid 10878) was used to generate shRNA constructs against NRG1. The 21-bp target sequences against NRG1 were CGTGGAATCAAACGAGATCAT and CCACAGAAGGAG CAAATACTT respectively. These two constructs were afterwards used to produce lentiviruses and establish stable the cell lines named as 'shNRG1-1' and 'shNRG1-2', respectively. For lentivirus production, the packaging plasmid psPAX2 and the envelope plasmid pMD2.G were kind gifts from Dr Yi Qin, Pancreatic Cancer Institute, Fudan University.

Lentivirus production and stable cell line selection. To produce lentiviral particles, the shRNA constructs were co-transfected with the psPAX2 and pMD2.G plasmids at a ratio of 4:3:1 into the 293T cells using Lipofectamine ${ }^{\mathrm{TM}} 2000$ Transfection Reagent (11668027; Thermo Fisher Scientific, Inc., Waltham, MA, USA). Lentiviral particles were harvested after $48 \mathrm{~h}$ of transfection. After being infected by the lentiviral particles, the K1 and TPC1 cells were selected by puromycin, respectively to obtain cell lines that stably expressed shRNA.

Flow cytometric analysis of ROS generation and intracellular glutathione (GSH) activity assay. An oxidant-sensitive fluorescent probe (DCFH-DA) was used to detect intracellular ROS levels (Sigma-Aldrich, St. Louis, MO, USA). Briefly, the cells were washed twice by phosphate-buffered saline (PBS), and were then stained with DCFH-DA $(10 \mu \mathrm{mol} / \mathrm{l})$ at $37^{\circ} \mathrm{C}$ for $20 \mathrm{~min}$ as per the manufacturer's instructions. DCFH-DA was deacetylated by intracellular non-specific esterase, and was then oxidized by ROS to generate the fluorescent compound, 2,7-dichlorofluorescein (DCF). The DCF fluorescence intensity was detected using a FACScan flow cytometer (BD Biosciences, San Jose, CA, USA). Intracellular GSH activity that reflects the oxidative status of cells was determined using the GSH/GSSG Ratio Detection Assay kit (ab138881; Abcam, Cambridge, MA, USA).

$R N A$ extraction and reverse transcription-quantitative $P C R$ (RT-qPCR). TRIzol reagent (10296010; Thermo Fisher Scientific, Inc.) was used to extract total RNA from the cells as per the manufacturer's instructions. cDNA reverse transcription was achieved using the PrimeScript ${ }^{\mathrm{TM}}$ RT Master Mix (RR036A; Takara Bio, Inc., Beijing, China). Relative quantitative (real-time) PCR was performed using SYBR ${ }^{\circledR}$ Premix Ex $\mathrm{Taq}^{\mathrm{TM}}$ II (Tli RNaseH Plus) (RR820Q; Takara Bio, Inc.) to determine the mRNA expression levels of candidate genes normalized to $\beta$-actin, using the ABI 7900HT Real-Time PCR system (Applied Biosystems, Waltham, MA, USA) by the standard protocol. The thermocycling conditions are as follows: step $1,50^{\circ} \mathrm{C}$ for $2 \mathrm{~min}$; step $2,95^{\circ} \mathrm{C}$ for $5 \mathrm{~min}$ (step 1 and step 2 are the hold stage); step $3,95^{\circ} \mathrm{C}$ for $10 \mathrm{sec}$; step 4 , $60^{\circ} \mathrm{C}$ for $1 \mathrm{~min}$ (recycle step 3 and step 4 to a total of 40 cycles, making the PCR stage); step $5,95^{\circ} \mathrm{C}$ for $15 \mathrm{sec}$; step $6,60^{\circ} \mathrm{C}$ for $1 \mathrm{~min}$; step $7,95^{\circ} \mathrm{C}$ for $15 \mathrm{sec}$ (step 5 to step 7 are the melt curve stage). All reactions were conducted in triplicate. Relative quantification $(\Delta \Delta \mathrm{Cq})$ was carried out as previously described (22). The primers used for PCR were as follows: $N R G 1$ forward, 5'-CTAACATAGGAGAGTTAGGTGGC-3' and reverse, 5'CTGTGGGCCAGTTAAACCTCTT-3'; ME1 forward, 5'-CCTCACTACTGCTGAGGTTATAGC-3' and reverse, 5'-CGGTTCAGGATAAACTGTGGCTG-3'; TXNRD1 forward,5'-GCAATCCAGGCAGGAAGATTGCT-3' and reverse, 5'-CTCTTGACGGAATCGTCCATTCC-3'; GCLC forward, 5'-GTGGTACTGCTCACCAGAGTG-3' and reverse, 5'-AGCTCCGTGCTGTTCTGGGCCTT-3'; GCLM forward, 5'-ATCTTGCCTCCTGCTGTGTGATGC-3' and reverse, 5'-CAATGACCGAATACCGCAGTAGCC-3'; HMOX1 forward, 5'-GCTCTGGAAGGAGCAAAATCAC ACC-3' and reverse, 5'-TATGACCCTTGGGAAACAAAG TCTGG-3'; NQO1 forward, 5'-AAGCCCAGACCAACTTCT-3' and reverse, 5'-GCGTTTCTTCCATCCTTC-3'; GAPDH forward, 5'-GAACGGGAAGCTCACTGG-3' and reverse, 5'-GCCTGCTTCACCACCTTCT-3'; and $\beta$-actin forward, 5'-CACCATTGGCAATGAGCGGTTC-3' and reverse, 5'-AGGTCTTTGCGGATGTCCACGT-3'.

Western blot analysis. Cell lysates were obtained from $1 \times 10^{6}$ cultured cells with a mixture of RIPA protein extraction reagent, protease inhibitor and phosphatase inhibitor (11836153001 and 4906845001; Roche, Shanghai, China). The protein concentration was determined by bicinchoninic acid assay (BCA). Equal amounts $(50 \mu \mathrm{g})$ of total protein lysate were separated by 5 to $10 \%$ SDS-PAGE and then transferred onto PVDF membranes. The membranes were then blocked in $5 \%$ non-fat milk at room temperature for $1 \mathrm{~h}$. Following this treatment, the membranes were probed with primary antibodies against NRG1, ERK1/2, NRF2, $\beta$-actin and GAPDH at $4^{\circ} \mathrm{C}$ overnight. Following incubation in a solution of goat anti-rabbit or anti-mouse IgG at room temperature for $1 \mathrm{~h}$, the membranes were washed using TBST and then treated with enhanced chemiluminescence reagents (Thermo Fisher Scientific, Inc.).

Cell proliferation assays. The Cell Counting kit-8 (CCK-8; CK04; Dojindo Laboratories, Shanghai, China) assay and plate clone formation assay were used to evaluate cell viability and the cell proliferative capability. Briefly, 1,000 cells were seeded in 1 well of a 96-well plate for detection for 5 days. At the same time each day, the cell culture medium was changed and CCK- 8 reagents were added at a ratio to medium of 1:10. Following $2 \mathrm{~h}$ of incubation, the optical density at $450 \mathrm{~nm}$ of each well was measured using a Synergy ${ }^{\mathrm{TM}} \mathrm{H} 4$ Hybrid Multi-Mode Microplate Reader (BioTek, Winooski, VT, USA) and transformed into cell numbers accordingly. For clone formation assay, 500 cells were plated into a well of a 6-well plate and cultured in a cell incubator for 14 days with regular medium changes. The cells were then washed with PBS and fixed with 4\% paraformaldehyde (P1110; Solarbio Biotechnology, Shanghai, China) at room temperature for $30 \mathrm{~min}$. Then cells were stained with crystal violet staining solution (0.5\%) (60506ES60; Yeasen Biotechnology, Shanghai, China) at room temperature for $30 \mathrm{~min}$ and then washed with PBS 3 times. The plate was dried at room temperature and images were aqcuired using a NEM-AL10 device developed by Honor, Huawei (Guangdong, China). Clone numbers were counted by Image $\mathbf{J}$ software and analyzed.

Statistical analysis. All statistical analyses were conducted using the SPSS software program (version 22.0; IBM Corp., Armonk, NY, USA). Pearson's $\chi^{2}$ test was conducted to assess the association between NRG1 expression and patient 
Table I. Association between NRG1 and lymph node metastasis in patients with thyroid cancer according to immunohistochemical analysis and patient outcomes.

\begin{tabular}{|c|c|c|c|c|}
\hline \multirow[b]{2}{*}{ Tumor tissue } & \multicolumn{2}{|c|}{ Regional lymph node metastasis ${ }^{\mathrm{a}}$} & \multicolumn{2}{|c|}{ Recurrence (local or distant) ${ }^{\mathrm{b}}$} \\
\hline & Negative & Positive & Negative & Positive \\
\hline \multicolumn{5}{|l|}{ NRG1 } \\
\hline- & $43(22.0)$ & $24(12.2)$ & $66(33.7)$ & $1(0.5)$ \\
\hline+ & $21(10.7)$ & $36(18.4)$ & $49(25)$ & $8(4.1)$ \\
\hline++ & $23(11.7)$ & $49(25.0)$ & $60(30.6)$ & $12(6.1)$ \\
\hline
\end{tabular}

Data are presented as number $(\mathrm{n})$ and the percentage in parentheses. ${ }^{\mathrm{a}}$ Pearson's $\chi^{2}$ test $=16.462, \mathrm{P}=0.001$; ${ }^{\mathrm{b}} \mathrm{Pearson}$ 's $\chi^{2}$ test $=9.280, \mathrm{P}=0.010$. NRG1, neuregulin 1.

outcomes (Table I) and between NRG1 expression and NRF2 expression in thyroid cancer samples (Table II). A value of $\mathrm{P}<0.05$ was considered to indicate a statistically significant difference. Pearson's correlation coefficient test was used to assess the correlation between the expression of NRG1 and the expression of NRF2 (Fig. 5). Values of $\mathrm{P}<0.05$ and $\mathrm{R} 2>0.1$ were considered to indicate statistically significant differences. One-way ANOVA with Dunnett's Multiple Comparison test was used to determine the statistical significance and P-values in Figs. 2-4 and a value of $\mathrm{P}<0.05$ was considered to indicate a statistically significant difference.

\section{Results}

$N R G 1$ is a potential prognostic marker of thyroid cancer. The clinicopathological characteristics of the patients have been provided in a previous study by our group (23). IHC staining and scoring was used to detect the protein levels of NRG1 in the PTC samples. The NRG1 level was higher in the PTC samples than that in the normal tissues (Fig. 1). In order to examine the role of NRG1 in the lymph node metastasis (LNM) of PTC, we examined its level in a PTC tissue microarray composed of 196 patient samples. Further analysis demonstrated that NRG1 was a positive indicator of PTC LNM at diagnosis (Table I).

$N R G 1$ regulates the viability and proliferation of thyroid cancer. To determine the biological role of NRG1 in thyroid cancer, we generated PTC cells K1 and TPC1) in which NRG1 was knocked down using shRNA (two shRNA constructs effectively decreased NRG1 expression. The knockdown efficiency was detected by RT-qPCR and western blot analysis after $48 \mathrm{~h}$ of transfection (Fig. 2A). To ascertain the contribution of NRG1 to the proliferation of PTC cells, a CCK-8 cell proliferation assay was conducted. Cell viability was significantly decreased when NRG1 was knocked down (Fig. 2B). Plate clone formation assay was also performed to detect the effect of NRG1 on thyroid cancer cell proliferation. The colony numbers of TPC1 and $\mathrm{K} 1$ cells were significantly decreased when NRG1 expression was silenced (Fig. 2C).

NRG1 regulates the ERK1/2 pathway and the expression of $N R F 2$. To explore the potential mechanisms through which NRG1 regulates the viability and proliferation of thyroid
Table II. Positive association between NRG1 and NRF2 in PTC tissues according to immunohistochemical analysis.

\begin{tabular}{lccc}
\hline & \multicolumn{3}{c}{ NRF2 } \\
\cline { 2 - 4 } Tumor tissue & - & + & ++ \\
\hline NRG1 & & $19(9.7)$ & $12(6.1)$ \\
- & $36(18.3)$ & $30(15.3)$ & $16(8.2)$ \\
+ & $11(5.6)$ & $26(13.3)$ & $37(18.9)$ \\
++ & $9(4.6)$ & 26 & \\
\hline
\end{tabular}

Data are presented as number (n) and the percentage in parentheses. Pearson's $\chi^{2}$ test $=40.307, \mathrm{P}=0.001$. NRG1, neuregulin $1 ; \mathrm{NRF} 2$, nuclear factor E2-related factor 2; PTC, papillary thyroid cancer.

cancer cells, we further examined the association between NRG1 and other thyroid cancer-related pathways. The results revealed that both the expression of ERK1/2 and NRF2 decreased when NRG1 was knocked down (Fig. 3A).

$N R G 1$ is essential for the transcription of NRF2 target genes. We further detected the downstream targets of the NRF2/ARE pathway to explore the biological effects of NGR1. The results of RT-qPCR revealed that NRG1 silencing decreased the expression of ARE-driven genes, including $G C L C, G C L M$, HMOX1, NQO1, ME1 and TXNRD (Fig. 3B and C). These results suggest that NRG1 is an upstream transcriptional regulator of antioxidant enzymes, whose regulatory function is dependent on the NRF2/ARE pathway.

NRG1 modulates the redox status of thyroid cancer cells. Subsequently, we attempted to explore the contribution of NRG1 to redox homeostasis. We found that when NRG1 was knocked down, the GSH/GSSG ratios in the PTC cells were significantly decreased, suggesting that NRG1 maintains the reductive status of cells (Fig. 4A and B). The intracellular ROS levels were examined to examine the effect of NRG1 on the oxidative stress level of PTC cells directly. Consistent with the changes observed in the GSH/GSSG ratio levels, the knockdown of NRG1 significantly elevated the intracellular ROS levels of the PTC cells, indicating that PTC cells 

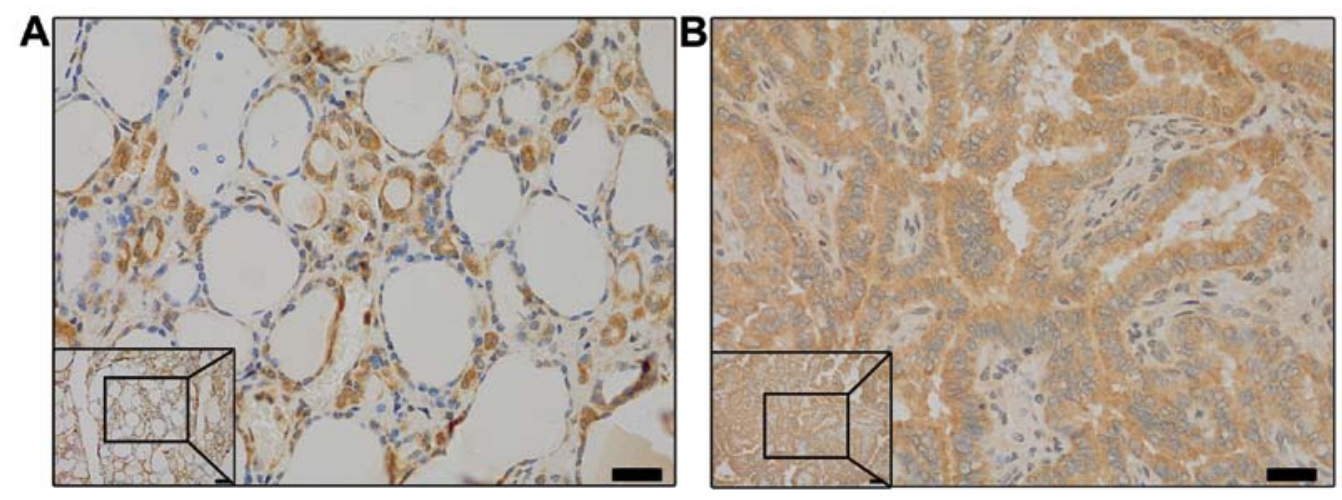

Figure 1. NRG1 protein staining indices by immunohistochemistry for NRG1 expression in thyroid cancer and adjacent normal tissues. NRG1 was expressed predominantly in the membrane and plasma of cells in the tumor and para-tumor regions. NRG1 was expressed weakly positive in (A) the para-tumor tissues and strongly positive in (B) the thyroid cancer regions. The cell images presented are enlarged versions of the area indicated in the box in the lower corner of the. The scale bars for the images inside the boxes and the enlarge image are 100 and $50 \mu \mathrm{m}$, respectively. NRG1, neuregulin 1.
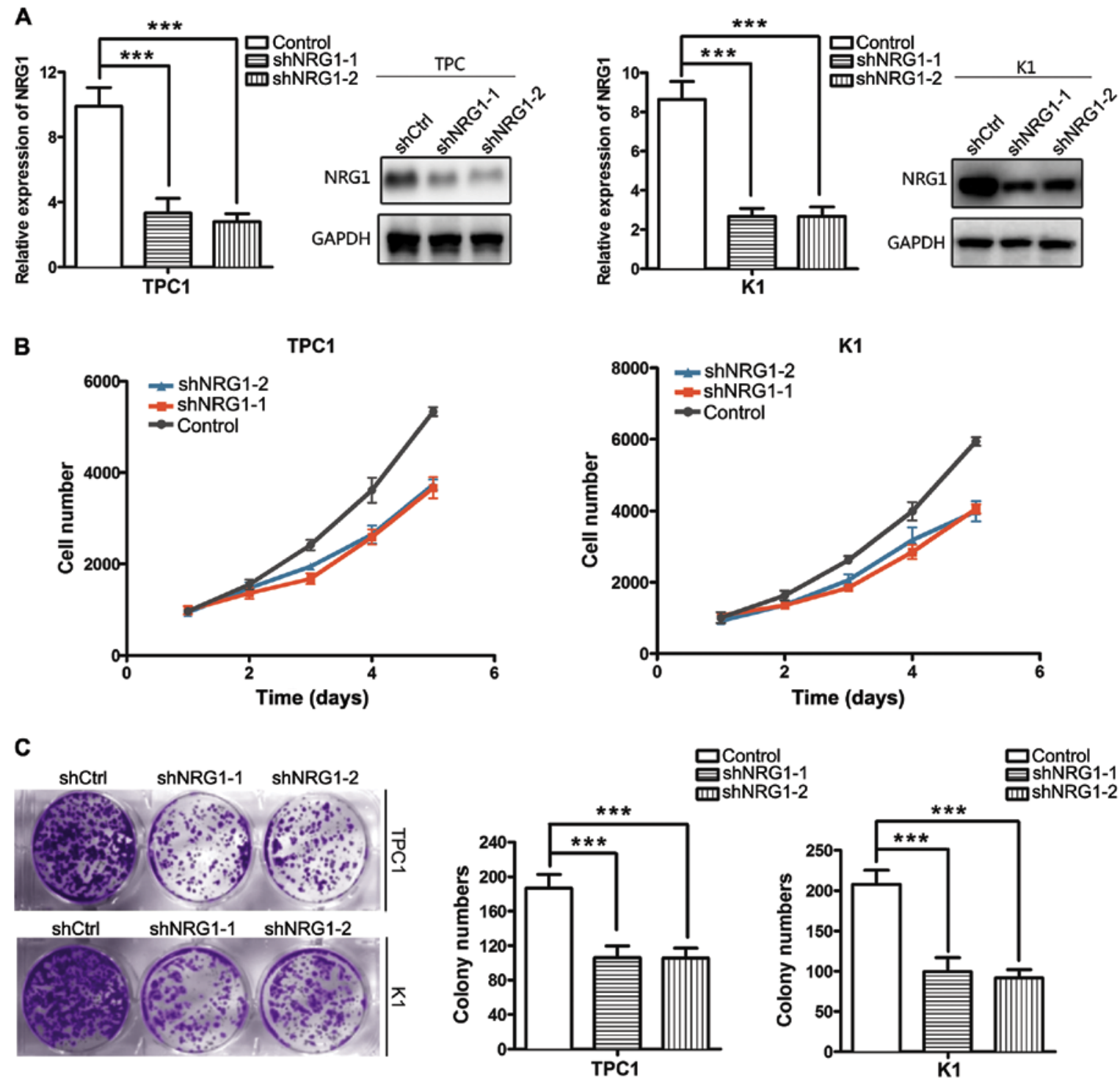

Figure 2. NRG1 promotes cell viability and proliferation. (A) Knockdown efficiency of NRG1, proved by quantitative real-time PCR and western blot analysis. (B) Cell viability was measured in thyroid cancer cells with depleted NRG1 expression $(n=6)$. (C) Plate clone formation assay when NRG1 was knocked down. ${ }^{* * * *} \mathrm{P}<0.001$. All experiments were repeated twice. NRG1, neuregulin 1.

in which NRG1 expression is knocked down are subjected to more severe oxidative stress (Fig. 4C and D). These results demonstrate that NRG1 plays an important regulatory role in the redox balance of PTC cells. 
A

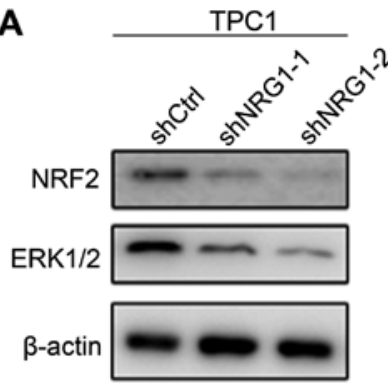

B

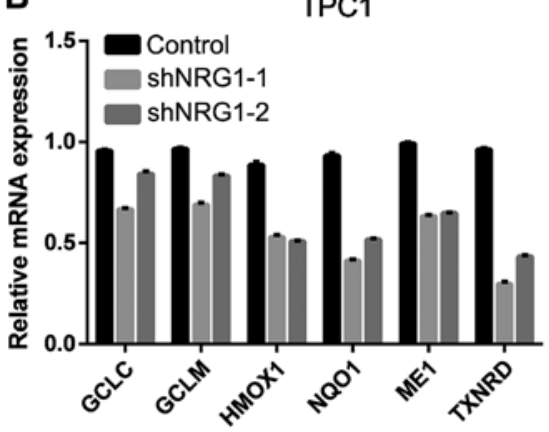

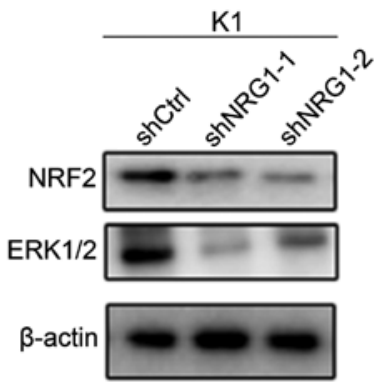

C

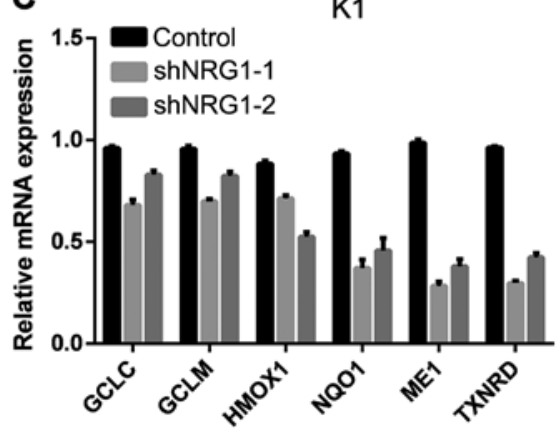

Figure 3. NRG1 regulates the NRF2/ARE pathway. (A) Knockdown of NRG1 downregulated NRF2 expression. (B and C) Knockdown of NRG1 decreased the expression of antioxidant genes, including GCLC, GCLM, HMOX1, NQO1, ME1 and TXNRD. All experiments were repeated twice. NRG1, neuregulin 1; NRF2, nuclear factor E2-related factor 2.

A

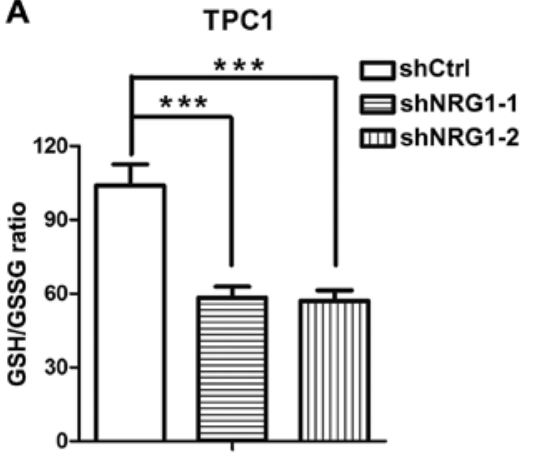

C

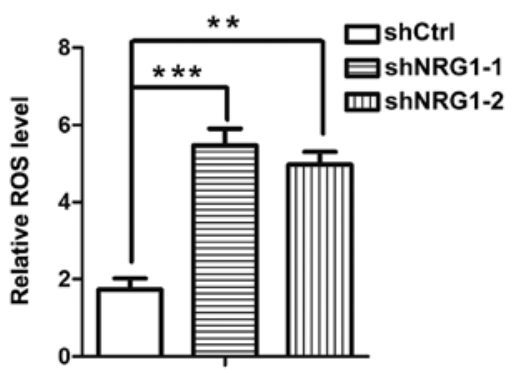

B

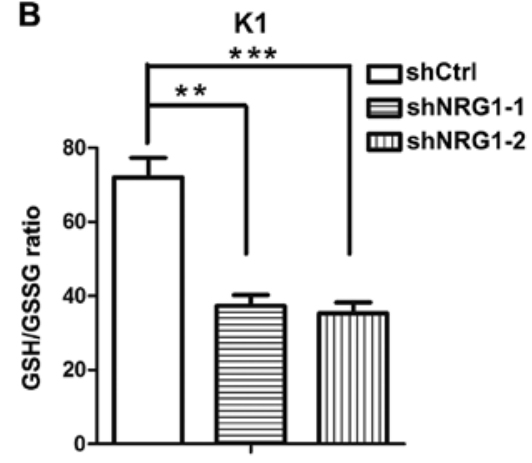

D

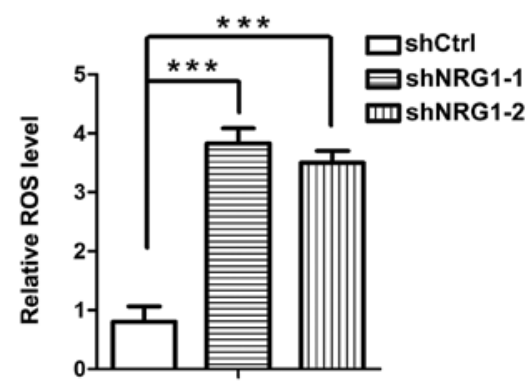

Figure 4. NRG1 maintains the intracellular redox status. (A and B) Intracellular GSH and GSSG levels were determined. NRG1 elevated the GSH/GSSG ratio in thyroid cancer cells $(\mathrm{n}=3)$. (C and D) Decreased NRG1 expression increased intracellular ROS levels $(\mathrm{n}=3)$. All $\mathrm{P}<0.01$. All experiments were repeated twice. ${ }^{* *} \mathrm{P}<0.01 ;{ }^{* * * *} \mathrm{P}<0.001$. NRG1, neuregulin 1; GSH, glutathione; GSSG, glutathione disulfide; ROS, reactive oxygen species.

NRG1 expression is positively associated with NRF2 expression in PTC samples. The association between NRG1 and NRF2 was analyzed by IHC staining of PTC tissues from 196 patients treated at the Department of Head and Neck Surgery, Fudan University Shanghai Cancer Center and by the analysis of data from 490 patient data extracted from TCGA database. In the 196 PTC samples, the NRG2 level was high, in parallel with the high level of NRG1 in the PTC samples. Statistical analysis indicated a positive association between NRG1 and NRG2 in the PTC tissue microarray (Table II). Linear regression analysis of the expression of NRG1 and NRF2 of 490 patients with PTC derived from the TCGA 


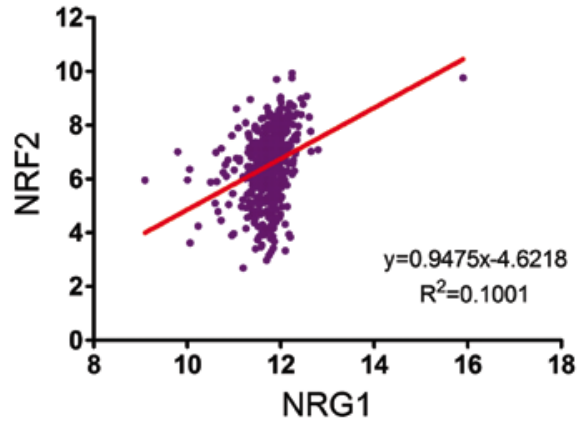

Figure 5. The expression of NRF2 positively correlates with that of NRG1; data were obtained from 490 patients with thyroid cancer from the The Cancer Genome Atlas database to detect the correlation between the expression of NRG1 and NRF2. Linear regression analysis of the expression data revealed a significant positive correlation between the expression of NRF2 and NRG1 $\left(\mathrm{R}^{2}=0.1001, \mathrm{P}<0.0001\right)$. NRG1, neuregulin 1; NRF2, nuclear factor E2-related factor 2.

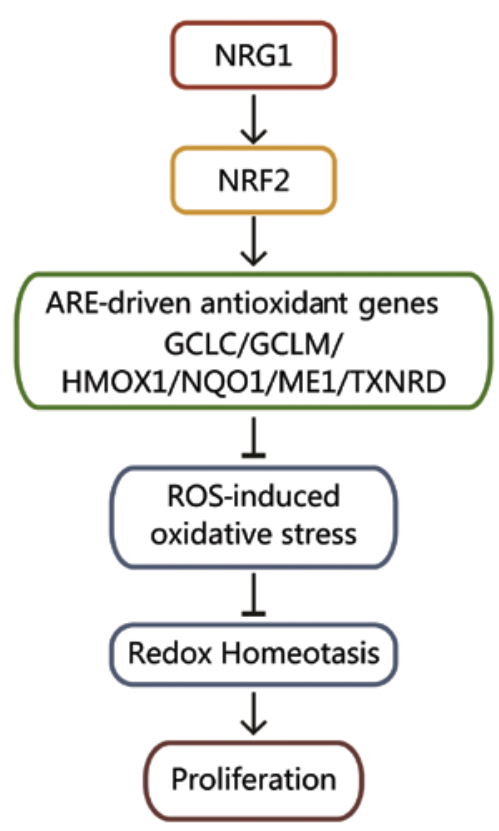

Figure 6. Schematic illustration of the mechanisms through which NRG1 regulates redox homeostasis in papillary thyroid cancer cells. NRG1 positively regulates NRF2, which upregulates ARE-related antioxidant genes (GCLC GCLM, HMOX1, NQO1, ME1 and TXNRD). Through these mechanisms, NRG1 reduces ROS-induced oxidative stress and maintains the moderate redox homeostasis that is essential for the proliferation of papillary thyroid cancer cells. NRG1, neuregulin 1; NRF2, nuclear factor E2-related factor 2; ROS, reactive oxygen species.

database revealed a similar pattern (Fig. 5). These results suggest a positive correlation between the expression of NRG1 and NRF2, implying the role of NRG1 in redox homeostasis clinically.

Mechanisms through which NRG1 regulates redox homeostasis in PTC cells. According to the results mentioned above, we explored the mechanisms through which NRG1 regulates redox homeostasis in PTC cells. NRG1 positively regulates NRF2, which upregulates ARE-related antioxidant genes (GCLC, GCLM, HMOXI, NQO1, MEl and TXNRD). Through these mechanisms, NRG1 reduces ROS-induced oxidative stress and maintains the moderate redox homeostasis that is essential for the proliferation of papillary thyroid cancer cells (Fig. 6).

\section{Discussion}

PTC is the most common thyroid cancer. PTC typically has a favorable prognosis, with an overall 10-year survival rate $>90 \%$. As epidemiological studies have reported, the incidence of thyroid cancer, particularly that of PTC, has markedly increased over the past decades worldwide $(1,24,25)$. In addition, the number of the patients with refractory thyroid cancer has also increased. In order to improve the prognosis of patients with PTC, it is of utmost importance to elucidate the biological mechanisms underlying the initiation and progression of PTC.

Cells can only live with intracellular homeostases to conduct their biological progresses, such as $\mathrm{pH}$ homeostasis, temperature homeostasis and redox homeostasis. In order to maintain intracellular homeostasis, cells manage to react rapidly to perturbations of redox homeostasis through a series of redox balancing mechanisms (26). ROS produced by oxygen metabolism are one of the perturbations to redox homeostasis. Excessive ROS productoin and ROS-mediated cell damage give rise oxidative stress in cells. Thus, cells under oxidative stress employ the antioxidant mechanism to survive and proliferate, and the NRF2/ARE signaling pathway plays a pivotal role in this process (27).

NRG1, a membrane glycoprotein, mediates cell-cell signaling and is essential for the development and growth of multiple organ systems. NRG1 can be processed into numerous isoforms, which allows it to perform a wide range of functions. NRG1 has been reported to interact with a number of important signaling pathways, including the ErbB pathway, Ras/MAPK/ERK1/2 pathway and PI3K pathway $(4,5,7,8)$.

According to the microarray analysis of the human genome chip in this study, NRG1 expression was upregulated in the PTC tissue samples compared with corresponding non-tumor thyroid tissue samples obtained at the Fudan University Shanghai Cancer Center. It was also revealed that NRG1 regulates the expression of NRF2 in PTC cells and in clinical tissue samples.

Downstream targets of the NRF2/ARE pathway are various antioxidant enzymes, whose expression levels protect cancer cells from oxidative stress (28). The expression levels of several antioxidant enzymes were examined in this study, including GCLC, GCLM, HMOX1, NQO1, ME1 and TXNRD. ME1 is a nicotinamide adenine dinucleotide phosphate (NADP)-dependent malic enzyme producing NADPH that can be used for antioxidation (29). TXNRD1, one of pyridine nucleotide oxidoreductases, also balances redox homeostais (30). Both GCLC and GCLM are biosynthetic enzymes of GSH, which participates in antioxidant reactions and redox homeostasis maintenance (31). HMOX1, acting as an essential enzyme in heme catabolism, can counteract inflammation by upregulating interleukin (IL)-10 and IL-1 receptor agonist IL-1 receptor agonist (IL-1RA) expression (32). NQO1 encodes NAD $(\mathrm{P}) \mathrm{H}$ dehydrogenase, which can protect cellular membranes from peroxidative injury (33).

In this study, we demonstrated that NRG1 may be a regulator of redox homeostasis, by promoting the transcription 
of these target antioxidant enzymes via NRF2. Due to the upregulation of these antioxidant enzymes by NRF2, intracellular ROS levels are regulated to moderate levels, creating a homeostasis that is essential for the survival, proliferation, migration and invasion for PTC cells.

In conclusion, in this study, we demonstrated that NRG1 activates the antioxidant pathway by upregulating the expression of antioxidant enzymes through NRF2, thus modulating redox homeostasis in PTC (Fig. 6). However, to clarify the exact mechanisms through which NRG1 activates the antioxidant response, further studies are warranted. The role of NRG1 in ROS detoxification suggests the potential use of NRG1 as a therapeutic target in the treatment of PTC.

\section{Acknowledgements}

Not applicable.

\section{Funding}

This study was supported by grants from the National Natural Science Foundation of China (no. 81702649 to NQ, nos. 81572622 and 81272934 to QHJ, nos. 81472498 and 81772851 to YLW and no. 81502317 to WJW) and from the Shanghai Rising-Star Program (no. 15QA1401100 to YLW).

\section{Availability of data and materials}

The analyzed datasets generated during the study are available from the corresponding author on reasonable request.

\section{Authors' contributions}

TTZ, NQ, GHS, YXZ and RLS were involved in the conception and design of the study. LZ, TTZ and YW were involved in the acquisition of the clinical data. NQ, LZ, WJW, YLW and QHJ conducted the experiments. YJW and XMM analyzed and interpreted the data. TTZ, NQ, LZ and RLS wrote the manuscript. All authors have read and approved the manuscript.

\section{Ethics approval and consent to participate}

This study was approved by the Human Ethics Committee/ Institutional Review Board of Fudan University Shanghai Cancer Center. All 196 patients signed the written informed consent.

\section{Consent for publication}

Not applicable.

\section{Competing interests}

The authors declare that they have no competing interests.

\section{References}

1. Lim H, Devesa SS, Sosa JA, Check D and Kitahara CM: Trends in thyroid cancer incidence and mortality in the United States, 1974-2013. JAMA 317: 1338-1348, 2017.
2. Haugen BR, Alexander EK, Bible KC, Doherty GM, Mandel SJ, Nikiforov YE, Pacini F, Randolph GW, Sawka AM, Schlumberger M, et al: 2015 American thyroid association management guidelines for adult patients with thyroid nodules and differentiated thyroid cancer: The American thyroid association guidelines task force on thyroid nodules and differentiated thyroid cancer. Thyroid 26: 1-133, 2016.

3. Acosta-Ortega J, Montalbán-Romero S, García-Solano J, SánchezSánchez C and Pérez-Guillermo M: Simultaneous medullary carcinoma of the thyroid gland and Hodgkin's lymphoma in bilateral lymph nodes of the neck: A potential pitfall in fine-needle aspiration cytology. Diagn Cytopathol 31: 255-258, 2004.

4. Falls DL: Neuregulins: Functions, forms, and signaling strategies. Exp Cell Res 284: 14-30, 2003.

5. Stefansson H, Steinthorsdottir V, Thorgeirsson TE, Gulcher JR and Stefansson K: Neuregulin 1 and schizophrenia. Ann Med 36: 62-71, 2004.

6. Wadugu B and Kühn B: The role of neuregulin/ErbB2/ErbB4 signaling in the heart with special focus on effects on cardiomyocyte proliferation. Am J Physiol Heart Circ Physiol 302: H2139-H2147, 2012.

7. Sheean ME, McShane E, Cheret C, Walcher J, Müller T, WulfGoldenberg A, Hoelper S, Garratt AN, Krüger M, Rajewsky K, et al: Activation of MAPK overrides the termination of myelin growth and replaces Nrg1/ErbB3 signals during Schwann cell development and myelination. Genes Dev 28: 290-303, 2014.

8. Cheng H, Terai M, Kageyama K, Ozaki S, McCue PA, Sato T and Aplin AE: Paracrine effect of NRG1 and HGF drives resistance to MEK inhibitors in metastatic uveal melanoma. Cancer Res 75: 2737-2748, 2015

9. Vyas S, Zaganjor E and Haigis MC: Mitochondria and cancer. Cell 166: 555-566, 2016.

10. Gorrini C, Harris IS and Mak TW: Modulation of oxidative stress as an anticancer strategy. Nat Rev Drug Discov 12: 931-947, 2013.

11. Sena LA and Chandel NS: Physiological roles of mitochondrial reactive oxygen species. Mol Cell 48: 158-167, 2012.

12. Favaro E, Bensaad K, Chong MG, Tennant DA, Ferguson DJ, Snell C, Steers G, Turley H, Li JL, Günther UL, et al: Glucose utilization via glycogen phosphorylase sustains proliferation and prevents premature senescence in cancer cells. Cell Metab 16: 751-764, 2012.

13. Diehn M, Cho RW, Lobo NA, Kalisky T, Dorie MJ, Kulp AN, Qian D, Lam JS, Ailles LE, Wong M, et al: Association of reactive oxygen species levels and radioresistance in cancer stem cells. Nature 458: 780-783, 2009.

14. Li W and Kong AN: Molecular mechanisms of Nrf2-mediated antioxidant response. Mol Carcinog 48: 91-104, 2009.

15. Taguchi K, Motohashi H and Yamamoto M: Molecular mechanisms of the Keap1-Nrf2 pathway in stress response and cancer evolution. Genes Cells 16: 123-140, 2011.

16. He H, Li W, Liyanarachchi S, Wang Y, Yu L, Genutis LK, Maharry S, Phay JE, Shen R, Brock P and de la Chapelle A: The role of NRG1 in the predisposition to papillary thyroid carcinoma. J Clin Endocrinol Metab: Nov 7, 2017 (Epub ahead of print).

17. Jendrzejewski J, Liyanarachchi S, Nagy R, Senter L, Wakely PE, Thomas A, Nabhan F, He H, Li W, Sworczak K, et al: Papillary thyroid carcinoma: Association between germline DNA variant markers and clinical parameters. Thyroid 26: 1276-1284, 2016.

18. Rogounovitch TI, Bychkov A, Takahashi M, Mitsutake N, Nakashima M, Nikitski AV, Hayashi T, Hirokawa M, Ishigaki K, Shigematsu K, et al: The common genetic variant rs944289 on chromosome 14q13.3 associates with risk of both malignant and benign thyroid tumors in the Japanese population. Thyroid 25: 333-340, 2015.

19. Amin MB, Edge S, Greene F, Byrd DR, Brookland RK, Washington MK, Gershenwald JE, Compton CC, Hess KR, Sullivan DC, et al (eds): AJCC Cancer Staging Manual. 8th edition. Springer, Chicago, IL, 2017.

20. Capes-Davis A, Theodosopoulos G, Atkin I, Drexler HG, Kohara A, MacLeod RA, Masters JR, Nakamura Y, Reid YA, Reddel RR and Freshney RI: Check your cultures! A list of cross-contaminated or misidentified cell lines. Int J Cancer 127: 1-8, 2010.

21. Ribeiro FR, Meireles AM, Rocha AS and Teixeira MR: Conventional and molecular cytogenetics of human non-medullary thyroid carcinoma: Characterization of eight cell line models and review of the literature on clinical samples. BMC Cancer 8: 371, 2008.

22. Livak KJ and Schmittgen TD: Analysis of relative gene expression data using real-time quantitative PCR and the 2(-Delta Delta C(T)) Method. Methods 25: 402-408, 2001. 
23. Shi RL, Qu N, Liao T, Wang YL, Wang Y, Sun GH and Ji QH: Expression, clinical significance and mechanism of Slit2 in papillary thyroid cancer. Int J Oncol 48: 2055-2062, 2016.

24. Chen W, Zheng R, Baade PD, Zhang S, Zeng H, Bray F, Jemal A, Yu XQ and He J: Cancer statistics in China, 2015. CA Cancer J Clin 66: 115-132, 2016.

25. La Vecchia C, Malvezzi M, Bosetti C, Garavello W, Bertuccio P, Levi F and Negri E: Thyroid cancer mortality and incidence: A global overview. Int J Cancer 136: 2187-2195, 2015.

26. Jaramillo MC and Zhang DD: The emerging role of the Nrf2-Keap1 signaling pathway in cancer. Genes Dev 27: 2179-2191, 2013.

27. Hayes AJ, Skouras C, Haugk B and Charnley RM: Keap1-Nrf2 signalling in pancreatic cancer. Int J Biochem Cell Biol 65 288-299, 2015.

28. Zhou S, Ye W, Shao Q, Zhang M and Liang J: Nrf2 is a potential therapeutic target in radioresistance in human cancer. Crit Rev Oncol Hematol 88: 706-715, 2013.

29. Jiang P, Du W, Mancuso A, Wellen KE and Yang X: Reciprocal regulation of p53 and malic enzymes modulates metabolism and senescence. Nature 493: 689-693, 2013

30. Dai B, Yoo SY, Bartholomeusz G, Graham RA, Majidi M, Yan S, Meng J, Ji L, Coombes K, Minna JD, et al: KEAP1-dependent synthetic lethality induced by AKT and TXNRD1 inhibitors in lung cancer. Cancer Res 73: 5532-5543, 2013.
31. Lien EC, Lyssiotis CA, Juvekar A, Hu H, Asara JM, Cantley LC and Toker A: Glutathione biosynthesis is a metabolic vulnerability in PI(3)K/Akt-driven breast cancer. Nat Cell Biol 18: 572-578, 2016.

32. Piantadosi CA, Withers CM, Bartz RR, MacGarvey NC, Fu P, Sweeney TE, Welty-Wolf KE and Suliman HB: Heme oxygenase-1 couples activation of mitochondrial biogenesis to anti-inflammatory cytokine expression. J Biol Chem 286: 16374-16385, 2011.

33. Ross D and Siegel D: NAD(P)H:quinone oxidoreductase 1 (NQO1, DT-diaphorase), functions and pharmacogenetics. Methods Enzymol 382: 115-144, 2004.

This work is licensed under a Creative Commons Attribution-NonCommercial-NoDerivatives 4.0 International (CC BY-NC-ND 4.0) License. 